\title{
Isolierung und Charakterisierung von zwei Hexokinasen unterschiedlicher Spezifität aus dem Flußkrebs Orconectes limosus (RAFINESQUE)
}

\author{
SABIne Wolter \\ II. Zoologisches Institut der Freien Universität Berlin
}

(Z. Naturforsch. 23 b, 839-845 [1968] ; eingegangen am 17. November 1967)

\begin{abstract}
Two hexokinase activities of different substrate specificity (fraction I and II) have been isolated by means of column chromatography on DEAE-cellulose from the antennal gland of the crayfish Orconectes limosus (RAFINESQUE). Chromatographically, the two fractions are uniform. Out of 10 sugars, fraction I converts mannose and fructose only; the phosphorylation of fructose amounts to $20 \%$ only of the conversion of mannose. Fraction II phosphorylates six out of the 10 sugars tested, and in its substrate specificity it corresponds to an unspecific hexokinase. The products of phosphorylation are with both enzymes for all substrates sugar-6-phosphates. The $K_{\mathrm{m}}$ values of fraction I for mannose and fructose are $3,9 \cdot 10^{-5}$ and $2,9 \cdot 10^{-3} \mathrm{M}$, those of fraction II for glucose, mannose and fructose $7,1 \cdot 10^{-5} \mathrm{M}, 8,0 \cdot 10^{-5} \mathrm{M}$ and $6,7 \cdot 10^{-3} \mathrm{M}$. The same enzymes have been found in the heart and in the hindgut, with proportions in the three organs, however, different.

Experiments with the crude extract had the following results as regards the properties of the enzymes: the activity is not particle-bound. Optimal activity is obtained at a $\mathrm{pH}$-value of more than 7,7 . High concentrations of salt as well as $\mathrm{pH}$-values below 6 or above 8 rapidly destroy the activity in storage.
\end{abstract}

Hexokinase-Aktivitäten wurden in einer Vielzahl von Organismen festgestellt ${ }^{1}$. Auf Grund ihrer Fähigkeit, entweder mehrere oder bevorzugt einen Zucker zu phosphorylieren, kann eine grobe Einteilung in spezifische und unspezifische Hexokinasen getroffen werden. Die am besten untersuchte unspezifische Hexokinase ist die kristallin erhaltene Hefehexokinase ${ }^{2}$, die von einer großen Anzahl als Substrate getesteter Hexosen neun Zucker in C-6-Stellung phosphoryliert ${ }^{3}$. $\mathrm{Zu}$ der Gruppe der unspezifischen Hexokinasen gehört ebenfalls die partikulär gebundene ${ }^{4}$, gut charakterisierte Hexokinase des Gehirns von Säugetieren, die im großen und ganzen die gleiche Substratspezifität wie die Hefehexokinase zeigt $^{5}$. Auch bei den Wirbellosen liegen Untersuchungen über unspezifische Hexokinase-Aktivitäten vor, so z. B. die Arbeiten über die Hexokinase-Akti-

1 Tabellarische Zusammenfassung bekannter Hexokinasen: R. K. Crane, in: P. D. Boyer, H. Lardy and K. Myrbäck, The Enzymes, Vol. 6, Academic Press, New York 1962, p. 47.

2 J. Berger, M. W. Slein, S. P. Colowick and C. F. Cori, J. gen. Physiol. 29, 379 [1946] ; M. Kunitz and M. R. McDonald, 29, 393 [1946]; K. Baily and E. C. WebB, Biochem. J. 42, 60 [1948].

3 A. Sols, G. Fuente, C. Villar-Palasi and C. Asensio, Biochim. biophysica Acta [Amsterdam] 30, 92 [1958].

4 R. K. Crane and A. Sols, J. biol. Chemistry 203, 273 [1953] ; M. K. Johnson, Biochem. J. 77, 610 [1960].

5 A. Sols and R. K. Crane, J. biol. Chemistry 210, 581 [1954].

6 M. Kerly and D. H. Leaback, Biochem. J. 67, 245 [1957].

7 A. N. Chatterjee, J. C. Ray and J. J. Grosh, Nature [London] 182, 109 [1958]. vität des Muskels der Wanderheuschrecke Locusta migratoria $^{6}$ und des Erregers der Schlafkrankheit Leishmania donovani ${ }^{7}$. Diese Enzyme wurden allerdings nicht hochgereinigt, da sie sehr instabil sind und beim Versuch einer Reinigung inaktiviert werden. Spezifische Hexokinase-Aktivitäten findet man in einigen Organen von Säugetieren, so z. B. die Fructo-1-kinase der Leber ${ }^{8}$, die einen vom Glucosestoffwechsel unabhängigen Umsatz der Fructose ermöglicht, und die Glucokinase der Leber ${ }^{9}$, die eine geringe Affinität für Glucose besitzt und hiermit zur Regulierung des Glucosestoffwechsels der Leber beiträgt. Bei den Wirbellosen sind spezifische Hexokinasen von dem Hundebandwurm Echinococcus granulosus $^{10}$ und dem Pärchenegel Schistosoma mansoni ${ }^{11}$ bekannt. Diese Tiere besitzen jeweils vier streng spezifische Hexokinasen für die Phosphory-

8 H. G. Hers, Biochim. biophysica Acta [Amsterdam] 8, 416 [1952]; H. G. Hers, in: S. P. Colowick and N. O. Kaplan, Methods in Enzymology, Vol.1, Academic Press, New York 1955, p. 286; R. E. Parks, E. Bengershom and H. A. Lardy, J. biol. Chemistry 227, 231 [1957].

9 D. L. Dipietro, C. Sharma and S. Weinhouse, Biochemistry 1, 455 [1962]; E. Vinuela, M. Salas and A. Sols, J. biol. Chemistry 238, PC 1175 [1963] ; D. G. Walker, Biochim. biophysica Acta [Amsterdam] 77, 209 [1963]; S. WeINhouse, V. Cristafalo, C. Sharma and H. P. Morris, in: G. Weber, Advances in Enzyme Regulation, Vol. 1, Pergamon Press, Oxford-London-Edinburgh-New York-ParisFrankfurt 1963, p. 363.

10 M. Agosin and L. Aravena, Biochim. biophysica Acta, 34, 90 [1959].

11 E. Bueding and J. Mackinnon, J. biol. Chemistry 215, 495 [1955]; E. Bueding, H. Ruppender and J. Mackinnon, Proc. nat. Acad. Sci. USA 40, 773 [1954]. 
lierung von Glucose, Mannose, Fructose oder Glucosamin.

Ziel der vorliegenden Untersuchungen war die Untersuchung der Hexokinase-Aktivität in den Organen eines Vertreters der decapoden Crustaceen, Orconectes limosus (Rafinesque), auf ihre Spezifität.

\section{Material und Methoden}

Die Versuchstiere stammten aus der Havel und wurden unter fließendem Leitungswasser bei einer sparsamen Erhaltungskost aus Fleisch, gekochten Kartoffeln und Mohrrüben gehalten. Für die Versuche wurden nur Weibchen verwendet. Nach Töten der Tiere in Chloroformdämpfen wurden die Organe mit der höchsten Hexokinase-Aktivität ${ }^{12}$ (Antennendrüse, Herz, Enddarm) entnommen und unter Kühlung in PotterElvehjem-Homogenisatoren mit Teflonstempel in einem Tris-EDTA-Medium (10 mM Tris-Puffer, $\mathrm{pH} 7,6,5 \mathrm{mM}$ Na-EDTA) homogenisiert. Es wurden immer Organe von mehreren Tieren vereinigt, für Versuche am Rohextrakt drei, für die Reinigungsversuche bis 150. Der durch Zentrifugieren bei $20000 \mathrm{~g}$ gewonnene Rohextrakt (Überstand) wurde weiter verwendet.

Die Hexokinase-Aktivität wurde durch den Zuckerverbrauch im Ansatz (Bestimmung des freien Zuckers vor und nach der Phosphorylierung) ${ }^{13}$ oder im gekoppelten optischen Test bestimmt. Der Ansatz für die erste Methode hatte folgende Zusammensetzung: TrisPuffer pH 8,0 $15 \mathrm{mM}$; Na-ATP $10 \mathrm{mM} ; \mathrm{MgCl}_{2}$ $50 \mathrm{mM}$; $\mathrm{KH}_{2} \mathrm{PO}_{4} 20 \mathrm{mM}$; Hexose $3 \mathrm{mM}$; Gesamtvolumen $0,5 \mathrm{ml}$. Die optimalen ATP- und $\mathrm{Mg}^{2 \oplus}$-Konzentrationen wurden in orientierenden Versuchen bestimmt. Der Zusatz von Phosphat diente zur Stabilisierung und Aktivitätserhöhung des Enzyms ${ }^{14}$. Gestartet wurde durch Zugabe von 0,5 ml Extrakt. In gleicher Weise wurden Proben ohne ATP inkubiert. Die Inkubationszeit betrug meist $15 \mathrm{~min}$, bei sehr verdünnten Extrakten maximal $30 \mathrm{~min}$, die Temperatur $25^{\circ} \mathrm{C}$. Die Reaktion wurde durch Zugabe von $2 \mathrm{ml} 0,3-n . \mathrm{Ba}(\mathrm{OH})_{2}$ und $2 \mathrm{ml} 5$-proz. $\mathrm{ZnSO}_{4}$-Lösung gestoppt. Hierbei wurden die gebildeten Zuckerphosphate mitgefällt. Nach $\mathrm{Zu}$ gabe von $5 \mathrm{ml} \mathrm{H}_{2} \mathrm{O}$ und zweimaliger Filtration (Filtrierpapier Schleicher \& Schüll 602 eh und $587 \mathrm{E}$ unter Zusatz von Hyflo-Supercel) wurde im Überstand der

12 R. Keller, Z. vgl. Physiol. 50, 119 [1965].

13 M. Somogyi, J. biol. Chemistry 160, 69 [1945]; R. K. Crane and A. Sols, in: S. P. Colowick and N. O. Kaplan, Methods in Enzymology, Vol. 1, Academic Press, New York 1955, p. 277.

14 Vgl. hierzu auch R. K. Crane and A. Sols, J. biol. Chemistry 203, 273 [1953]; H. Tiedemann u. J. Born, Z. Naturforschg. 14 b, 477 [1959].

15 Zit. nach K. Hinsberg u. K. Lang, Medizinische Chemie, Urban \& Schwarzenberg, München 1957, S. 352 u. 358.

16 Zit. nach G. Ashwell, in: S. P. Colowick and N. O. KAPLAN, Methods in Enzymology, Vol. 3, Academic Press, New York 1957, p. 73. freie Zucker bestimmt und aus der Differenz zwischen den Proben mit und ohne ATP der Zuckerverbrauch berechnet. Es wurden Dreifachbestimmungen ausgeführt. Die freien Zucker wurden nach folgenden Methoden bestimmt: Glucose, Mannose, Glucosamin, Galaktose, Galaktosamin und Ribose nach Nelson ${ }^{15}$; Fructose nach RoE ${ }^{15}$ mit Resorcin-HCl; 2-Desoxyglucose nach Dische ${ }^{16}$ mit Diphenylamin $-\mathrm{H}_{2} \mathrm{SO}_{4} ; N$-Acetylglucosamin nach Aminoff u.a. ${ }^{17}$ mit $p$-Dimethylaminobenzaldehyd; Fucose nach Dische und ShetrLES ${ }^{16}$ mit Cystein- $\mathrm{H}_{2} \mathrm{SO}_{4}$.

Aktivitätsbestimmungen im optischen Test wurden für die Hexosen Glucose, Mannose und Fructose durchgeführt. Für Glucose wurde die Phosphorylierung mit der Glucose-6-phosphatdehydrogenase-Reaktion gekoppelt 12, 18, für Fructose mit Phosphoglucoseisomerase und G-6-P-DH ${ }^{19}$, für Mannose mit Pyruvatkinase und Lactatdehydrogenase ${ }^{20}$. Die Extinktionsänderung wurde bei $366 \mathrm{~m} \mu$ und $25^{\circ} \mathrm{C}$ im Photometer Eppendorf gemessen und mit einem Kompensationsschreiber der Fa. Philips/Hamburg mit Linearisierungsglied registriert.

Der Proteingehalt der untersuchten Extrakte wurde nach Lowry u. a. ${ }^{21}$ bestimmt.

Zur Trennung der Aktivitäten durch Säulenchromatographie wurde DEAE-Cellulose der Fa. Serva/Heidelberg benutzt. Andere Ionenaustauscher (CM-Cellulose, Hydroxylapatit-Cellulose, DEAE-Sephadex) erbrachten keine Auftrennung der Enzymaktivität. Die Abmessungen der benutzten Säulen betrugen je nach Verwendungszweck $15 \cdot 160,15 \cdot 300$ oder $30 \cdot 300 \mathrm{~mm}$. Die Säulen wurden in einen Kühlmantel eingesetzt, durch den während der Chromatographie Wasser von $4-6{ }^{\circ} \mathrm{C}$ zirkulierte. Der Durchfluß durch die Säule wurde mit einer Desaga-Dosierpumpe reguliert. Als Versuchsmaterial diente der Überstand des Homogenats bei $20000 \mathrm{~g}$. Eluiert wurde nacheinander mit folgenden Lösungen und Gradienten: 1. Vorlauf: Tris-Puffer $50 \mathrm{mM}+\mathrm{Na}$-EDTA $5 \mathrm{mM}, \mathrm{pH} 7,8 ; 2$. kontinuierlicher linearer $\mathrm{pH}$-Gradient: Tris-Acetat-Puffer $50 \mathrm{mM}+$ Na-EDTA 5 mM, pH 6,3 gegen Puffer unter 1.; 3. kontinuierlicher linearer NaCl-Gradient $0 \rightarrow 1 \mathrm{M}$ : gepufferte NaCl-Lösung, $1 \mathrm{M}$ gegen Puffer unter 2. Die Fraktionen wurden in vorgegebenen Zeitintervallen mit dem Fraktionensammler der Fa. Serva/Heidelberg aufgefangen. Der Proteingehalt, der $\mathrm{pH}$-Wert und die Menge an zugesetztem Salz im Eluat wurden kontinuierlich be-

17 Zit. nach T. Ploetz, in: Hoppe-Seyler-Thierfelder, Handbuch der physiologisch- und pathologisch-chemischen Analyse, Bd. III/1, Springer, Berlin-Göttingen-Heidelberg 1955 , S. 646.

18 K. Thielmann u. E. Blume, Hoppe-Seylers Z. physiol. Chem. 328, 164 [1962].

19 H. U. Bergmeyer, Methoden der enzymatischen Analyse, Verlag Chemie, Weinheim 1962, S. 156.

20 H. Mattenheimer, Mikromethoden für das klinisch-chemische und biochemische Laboratorium, de Gruyter \& Co., Berlin 1961, S. 51.

21 Zit. nach R. W. Cowgill and A. B. Pardee, Experiments in biochemical research techniques, Wiley \& Sons, New York 1957, p. 176. 
stimmt (LKB-Uvicord, 254 m $\mu$; Schott-Meßelektroden 9516 gegen 9818 und Meßverstärker Knick pH 71; Leitfähigkeitsmeßgerät Fa. Pusl/München, Meßzelle PR 9513 Fa. Philips). Die einzelnen Fraktionen wurden auf Hexokinase-Aktivität durch den Zuckerverbrauch im Ansatz untersucht. Als Substrat diente ein Gemisch aus Glucose, Mannose und Fructose mit Endkonzentrationen von je $1,5 \mathrm{mM}$.

Die Identifizierung der Endprodukte der Phosphorylierung wurde nach LANGE und KoHN ${ }^{22}$ durchgeführt. Der Extrakt $(0,5 \mathrm{ml})$ wurde mit dem zu testenden Zukker $2 \mathrm{~h}$ bei $25{ }^{\circ} \mathrm{C}$ in einem Ansatz folgender Endkonzentration inkubiert: Tris-Puffer $\mathrm{pH} 8,015 \mathrm{~m} \mathrm{M}$; $\mathrm{MgCl}_{2} 50 \mathrm{mM}$; ATP $10 \mathrm{mM}$; Zucker $6 \mathrm{mM}$. Nach Stoppen der Reaktion mit $2 \mathrm{ml}$ absolutem Alkohol wurden Proben der Lösung auf säuregewaschenem Chromatographiepapier (Schleicher \& Schüll 2043 b gew.) aufsteigend mit Äthylacetat: Pyridin: Wasser = 10:4:3 (v/v) 2 h chromatographiert. Zur Entwicklung der luftgetrockneten Chromatogramme wurden die folgenden Reagenzien nacheinander aufgesprüht:

1. $10 \mathrm{mM} \mathrm{FeCl}_{3}$, danach $10 \mathrm{mM} \mathrm{K}_{4}\left[\mathrm{Fe}(\mathrm{CN})_{6}\right]$-Lösung ${ }^{23}$. Die am Startpunkt liegenden Phosphate erschienen als weiße Flecken auf blauem Untergrund.

2. Anilinphthalatreagenz auf reduzierende Zucker ${ }^{24}$. Die abgetrennten freien Zucker und die am Startpunkt liegenden Zucker-6-Phosphate erschienen als braune Flecke. Handelt es sich bei dem Phosphorylierungsprodukt um ein Zucker-1-Phosphat, so bleibt der Phosphatfleck weiß.

Zu Mittelwerten aus drei oder mehr Messungen wurde die Standardabweichung berechnet. Unterschiede wurden mit dem $t$-Verfahren auf statistische Signifikanz untersucht ${ }^{25}$.

\section{Ergebnisse}

Am Rohextrakt wurden orientierende Versuche vorgenommen, um einige Eigenschaften des Enzyms festzustellen. Meist wurde hierzu die Antennendrüse (das ganze Organ mit Ausnahme der Harnblase) als Enzymquelle verwendet. Zwischen $\mathrm{pH} 7$ und $\mathrm{pH} 9$ wurde eine $\mathrm{pH}$-Aktivitätskurve aufgenommen mit dem Ergebnis, daß der $\mathrm{pH}$-Wert zwischen $\mathrm{pH} 7,7$ und 9,0 keinen Einfluß auf die Hexokinase-Aktivität hat. Bei pH 7,2 erhält man nur noch $1 / 3$ der maximalen Aktivität. Die Hexokinase-Aktivität in den drei untersuchten Organen ist nicht partikelgebunden. Nach Zentrifugieren bei 20000 und $100000 \mathrm{~g}$ fand sich die gesamte Aktivität im Überstand (Tab. 1). Bei Lagerungsversuchen, in denen der Einfluß des $\mathrm{pH}$-Wertes und der Ionenstärke im Extrakt auf die Stabilität des Enzyms untersucht wurde,

22 C. F. Lange and P. Kohn, J. biol. Chemistry 236, 1 [1961].

23 Zit. nach H. Hettler, Chromat. rev. 1, 225 [1959].

\begin{tabular}{lcc}
\hline \multicolumn{1}{c}{ Organ } & \multicolumn{2}{c}{ Anteil an der } \\
& \multicolumn{2}{c}{ Gesamtaktivität [\%] } \\
& Uberstand & Sedimente \\
\hline Antennendrüse & 98,9 & 1,1 \\
Enddarm & 98,6 & 1,4 \\
Herz & 98,5 & 1,5 \\
\hline
\end{tabular}

Tab. 1. Verteilung der Hexokinase-Aktivität in der Zelle. Zentrifugiert: Homogenat $20 \mathrm{~min}$ bei $20000 \mathrm{~g}$. Überstand $60 \mathrm{~min}$ bei $100000 \mathrm{~g}$.

zeigte es sich, daß die Aktivität leicht zerstört wird. Einzelne Proben des Rohextraktes der Antennendrüse wurden mit $\mathrm{Na}(\mathrm{OH})$ oder $\mathrm{HCl}$ auf den gewünschten $\mathrm{pH}$-Wert oder mit gepufferten NaCl-Lösungen auf die gewünschte Salzkonzentration gebracht und $2 \mathrm{~h}$ bei $25^{\circ} \mathrm{C}$ oder einen Tag bei 2 bis $4{ }^{\circ} \mathrm{C}$ gelagert. Der teilweise Verlust der Aktivität bei pH-Werten unter 6 bzw. über 8 und bei hohen Salzkonzentrationen ist aus Abb. 1 ersichtlich. An

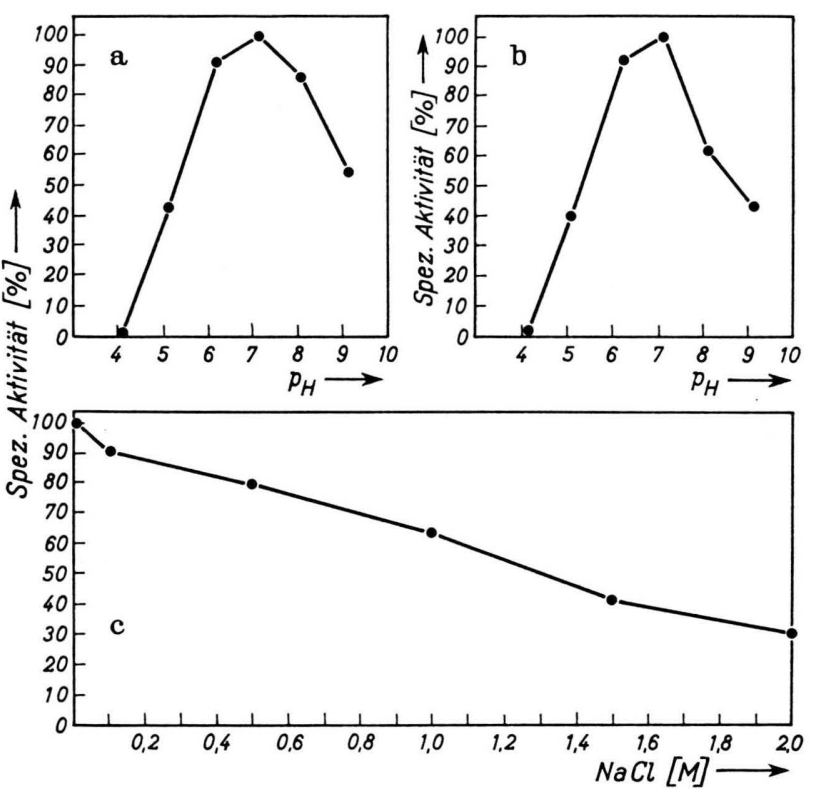

Abb. 1. Einfluß des pH-Wertes und der Ionenstärke im Extrakt auf die Stabilität der Enzymaktivität (Rohextrakt der Antennendrüse, Aktivitätsbestimmung im optischen Test mit Glucose als Substrat), restliche Aktivität nach Lagerung bei verschiedenem $\mathrm{pH}$ bzw. verschiedener Ionenstärke (\% Ausgangsaktivität). a) Lagerung bei verschiedenem $\mathrm{pH}$ ( $2 \mathrm{~h}$ bei $\left.25^{\circ} \mathrm{C}\right)$, b) Lagerung bei verschiedenem $\mathrm{pH}(24 \mathrm{~h}$ bei 2 bis $\left.4^{\circ} \mathrm{C}\right)$, c) Lagerung bei verschiedener Ionenstärke, $\mathrm{pH} \mathrm{7,2}$ $\left(21 \mathrm{~h}\right.$ bei $\left.2-4^{\circ} \mathrm{C}\right)$.

24 E. Stahl, Dünnschicht-Chromatographie, Springer, BerlinGöttingen-Heidelberg 1962, S. 498.

25 A. Linder, Planen und Auswerten von Versuchen, Birkhäuser-Verlag, Basel-Stuttgart 1959. 
vier verschiedenen Rohextrakten ( $\mathrm{pH} 7,2$ bis 7,4) und einer durch Chromatographie an DEAE-Cellulose gewonnenen Präparation der Antennendrüse wurde die Inaktivierung während der Lagerung bei $2-4{ }^{\circ} \mathrm{C}$ über längere Zeit verfolgt. Die fünf Proben wurden trotz gleicher Behandlung unterschiedlich schnell inaktiviert. Welche Faktoren hierfür verantwortlich sind, ist unklar. Die Aktivitätsbestimmung für die bisher beschriebenen Versuche erfolgte im optischen Test mit Glucose als Substrat. Die Phosphorylierung von sechs Zuckern (Glucose, Mannose, Fructose, Galaktose, Glucosamin, 2-Desoxyglucose) durch die Rohextrakte der drei Organe ergab keine signifikanten Differenzen der relativen Aktivitäten gegenüber verschiedenen Substraten, erbrachte also noch keinen Hinweis auf das Vorliegen mehrerer Enzyme. Mannose wurde in allen drei Organen am stärksten umgesetzt.

Eine Anreicherung der Hexokinase-Aktivität durch $\left(\mathrm{NH}_{4}\right)_{2} \mathrm{SO}_{4}$-Fällung scheiterte an der Inaktivierung des Enzyms ${ }^{26}$. Aus diesem Grunde wurde der Rohextrakt ohne weitere Vorreinigung der Chromatographie an Ionenaustauschern unterworfen. Nach Chromatographie an DEAE-Cellulose ergab sich nach der Aktivitätsbestimmung an den einzelnen Fraktionen mit dem Zuckergemisch aus Glucose, Mannose und Fructose eine Auftrennung in zwei aktive Fraktionen (Abb. 2). Die eine wurde im Vorlauf erhalten (Fraktion I), die andere im NaCl-Gradienten (Fraktion II). Letztere wurde sogleich an Sephadex G 25 entsalzt, um eine zu rasche Inaktivierung zu verhindern. Die beiden aktiven Fraktionen wurden auf ihre Aktivität gegen einzelne Zucker geprüft - es wurden wieder Glucose, Mannose und
Fructose verwendet - und es ergaben sich für die Antennendrüse für die relativen Umsatzraten von Glucose, Mannose und Fructose die in Tab. 2 dargestellten Verhältnisse. Bei der Rechromatographie der beiden Fraktionen im gleichen System erschienen

\begin{tabular}{|c|c|c|c|}
\hline \multirow[t]{2}{*}{$\begin{array}{l}\text { Nr. des } \\
\text { Versuchs }\end{array}$} & \multirow[t]{2}{*}{ Rohextrakt } & \multicolumn{2}{|c|}{$\begin{array}{l}\text { Chromatographie an DEAE- } \\
\text { Cellulose }\end{array}$} \\
\hline & & Fraktion I & Fraktion II \\
\hline $\begin{array}{l}1 \\
2 \\
3 \\
4\end{array}$ & $\begin{array}{l}1: 0,65: 0,28 \\
1: 0,54: 0,29\end{array}$ & $\begin{array}{l}1: 0,05: 0,13 \\
1: 0,02: 0,25 \\
1: 0,00: 0,18 \\
1: 0,01: 0.22\end{array}$ & $\begin{array}{l}1: 0,72:- \\
1: 1,20: 0,41 \\
1: 0,88: 0,49 \\
1: 0,99: 0,69\end{array}$ \\
\hline Mittel & $1: 0,60: 0,28$ & $1: 0,02: 0,20$ & $1: 0,95: 0,53$ \\
\hline
\end{tabular}

Tab. 2. Relative Umsatzraten für Mannose, Glucose und Fructose im Rohextrakt und in den durch Chromatographie an DEAE-Cellulose erhaltenen aktiven Fraktionen der Antennendrüse. Verhältniszahlen: Mannose: Glucose: Fructose.

die beiden Hexokinasen einheitlich an der erwarteten Stelle und zeigten unveränderte Aktivitätsverhältnisse. Beide Enzymaktivitäten findet man auch in Herz und Enddarm; sie zeigen die gleichen relativen Umsatzraten für Mannose und Glucose wie die Enzyme der Antennendrüse (Tab. 3).

Zur Spezifitätsuntersuchung wurde der Rohextrakt von 92 Antennendrüsen an DEAE-Cellulose chromatographiert, beide Fraktionen im gleichen System rechromatographiert und an beiden Fraktionen die Aktivität gegen 10 Zucker, darunter eine Pentose, über den Zuckerverbrauch im Ansatz bestimmt. Die Inkubationszeit betrug 30 min, da die Extrakte ziemlich verdünnt waren. Für jeden Zucker wurden 4 Bestimmungen ausgeführt. Die Ergebnisse zeigt Tab. 4 .

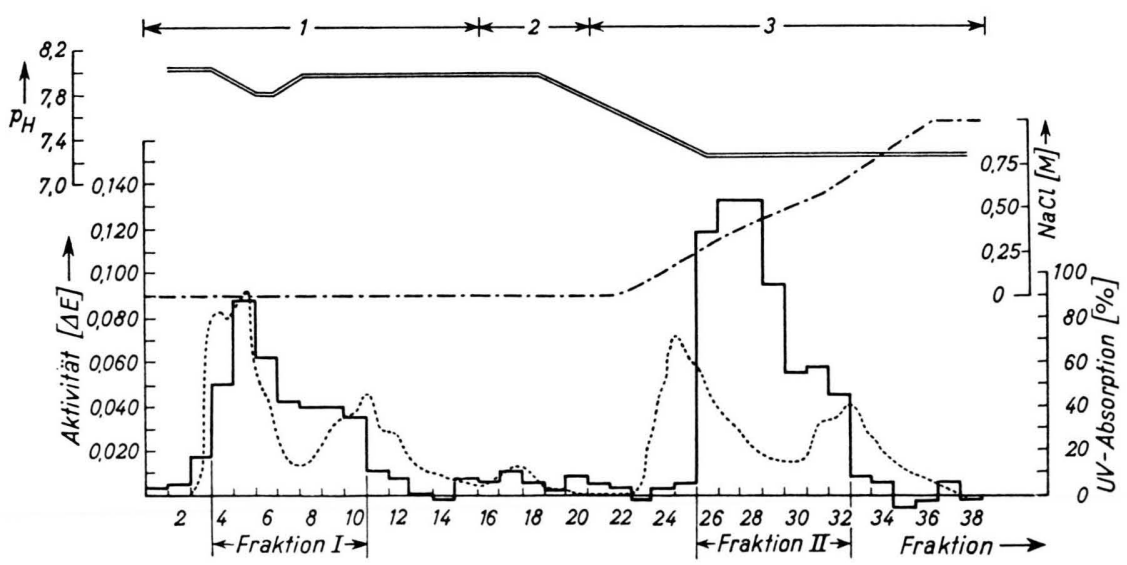

Abb. 2. Chromatographie des Rohextraktes von 20 Antennendrüsen an DEAE-Cellulose (Vers. Nr. 1 aus Tab. 2). Säule $15 \cdot 160 \mathrm{~mm}$. Fraktionen: $7 \mathrm{ml}$, - Aktivität, bestimmt mit einem Gemisch aus Glucose, Mannose, Fructose als Substrat, - - - Protein, bestimmt als Absorptionsänderung bei $254 \mathrm{~m} \mu$,

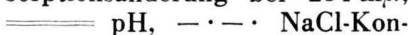
zentration, 1 Vorlauf, 2 kontinuierlicher, linearer pH-Gradient, 3 kontinuierlicher, linearer NaCl-Gradient.

\footnotetext{
26 Vgl. hierzu auch l. c. ${ }^{6}$.
} 


\begin{tabular}{|c|c|c|c|c|c|c|c|c|c|}
\hline \multirow{3}{*}{ Organ } & \multicolumn{6}{|c|}{ Spezifische Aktivität [nMol/mg Protein $\cdot \min ]$} & \multicolumn{3}{|c|}{$\begin{array}{c}\text { Relative Rate } \\
\text { Mannose: Glucose }\end{array}$} \\
\hline & \multicolumn{2}{|c|}{ Rohextrakt } & \multicolumn{2}{|c|}{ Fraktion I } & \multicolumn{2}{|c|}{ Fraktion II } & & Fraktion & Fraktion \\
\hline & Mannose & Glucose & Mannose & Glucose & Mannose & Glucose & extrakt & I & II \\
\hline $\begin{array}{l}\text { Antennen- } \\
\text { drüse }\end{array}$ & $186 \pm 7$ & $121 \pm 3$ & $678 \pm 41$ & \pm 15 & $462 \pm 10$ & $330 \pm 16$ & $\begin{array}{l}1: 0,65 \\
1: 0,60\end{array}$ & $\begin{array}{l}1: 0,05 \\
1: 0,02\end{array}$ & $\begin{array}{l}1: 0,72 \\
1: 0,95\end{array}$ \\
\hline Enddarm & $97,8 \pm 1,9$ & $50,4 \pm 1,9$ & $28,5 \pm 4,2$ & 0 & $62,7 \pm 1,5$ & $56,3 \pm 1,6$ & $1: 0,52$ & $1: 0$ & $1: 0,90$ \\
\hline Herz & $89,0 \pm 4,9$ & $57,3 \pm 8,2$ & $28,8 \pm 3,9$ & $0,63 \pm 2,7$ & $58,1 \pm 2,5$ & $50,4 \pm 2,0$ & $1: 0,64$ & $1: 0,02$ & $1: 0,87$ \\
\hline
\end{tabular}

Tab. 3. Relative Umsatzrate für Glucose und Mannose in den beiden Fraktionen der DEAE-Cellulose-Chromatographie für die drei untersuchten Organe. Der Umsatz von Mannose wurde gleich 1 gesetzt. Die Werte in der zweiten Zeile für die Antennendrüse sind Mittelwerte aus 4 Chromatographien (s. Tab. 2). Für die übrigen Werte wurden jeweils 3 Aktivitätsbestimmungen am gleichen Extrakt ausgeführt.

\begin{tabular}{|c|c|c|c|c|c|c|}
\hline \multirow[b]{2}{*}{ Zucker } & \multicolumn{3}{|c|}{ Fraktion I } & \multicolumn{3}{|c|}{ Fraktion II } \\
\hline & $\begin{array}{c}\text { Spez. Aktivität } \\
{[\mathrm{nMol} / \mathrm{mg} \text { Protein } \cdot \min ]}\end{array}$ & $\begin{array}{c}\text { Relative Rate } \\
\text { (bezogen auf } \\
\text { Mannose) }\end{array}$ & $P$ & $\begin{array}{c}\text { Spez. Aktivität } \\
{[\mathrm{nMol} / \mathrm{mg} \text { Protein } \cdot \min ]}\end{array}$ & $\begin{array}{c}\text { Relative Rate } \\
\text { (bezogen auf } \\
\text { Mannose) }\end{array}$ & $P$ \\
\hline Mannose & $447,0 \pm 19,8$ & 1,00 & 0,0002 & $123,0 \pm 12,3$ & 1,00 & 0,0002 \\
\hline Glucose & $6,6 \pm 26,8$ & $\overline{0,01}$ & 0,5 & $122,0 \pm 9,0$ & $\overline{0,99}$ & 0,0002 \\
\hline Galaktose & $29,2 \pm 23,0$ & 0,06 & 0,095 & $5,4 \pm 10,8$ & $\overline{0,04}$ & 0,084 \\
\hline Fructose & $99,1 \pm 23,0$ & 0,22 & 0,003 & $84,5 \pm 11,0$ & 0,69 & 0,0002 \\
\hline Glucosamin & $16,6 \pm 33,0$ & $\overline{0,04}$ & 0,3 & $78,0 \pm 15,0$ & $\overline{0,63}$ & 0,00024 \\
\hline $\begin{array}{l}N \text {-Acetyl- } \\
\text { glucosamin }\end{array}$ & $-23,1 \pm 15,0$ & $-0,05$ & 0,052 & $1,4 \pm 2,8$ & $\overline{0,01}$ & 0,33 \\
\hline 2-Desoxyglucose & $20,7 \pm 37,0$ & 0,05 & 0,135 & $114,0 \pm 14,5$ & 0,93 & 0,0002 \\
\hline Fucose & $11,4 \pm 34,0$ & 0,03 & 0,17 & $2,1 \pm 10,0$ & 0,02 & 0,21 \\
\hline Galaktosamin & $25,6 \pm 28,0$ & 0,06 & 0,14 & $27,5 \pm 9,0$ & 0,22 & 0,0013 \\
\hline Ribose & $24,9 \pm 30,0$ & 0,06 & 0,205 & $18,0 \pm 9,0$ & $\overline{0,15}$ & 0,045 \\
\hline
\end{tabular}

Tab. 4. Spezifität der Fraktionen I und II gegenüber 10 Zuckern (Antennendrüse). Anzahl der Messungen je Zucker: $n=4$. Die unterstrichenen Werte sind signifikant von Null verschieden $(P<0,01)$.

Fraktion I ist nur aktiv mit Mannose und Fructose; Mannose wird sehr viel stärker umgesetzt. Für Fraktion II sind 6 der 10 getesteten Zucker Substrate. Mit Hilfe der Papierchromatographie wurde die Natur der gebildeten Zuckerphosphate bestimmt. Es ergab sich, daß alle Zucker, die als Substrate dienen, von beiden Enzymen in C-6-Stellung phosphoryliert werden. Zur Bestimmung der Michaeli s-Konstanten der beiden Enzyme für Mannose, Glucose

\begin{tabular}{lcc}
\hline & \multicolumn{2}{c}{ M i c h a e li s - Konstante } \\
Substrat & \multicolumn{1}{c}{$\begin{array}{l}\text { Fraktion I } \\
\end{array}$} & Fraktion II \\
\hline Glucose & - & $7,1 \cdot 10^{-5}$ \\
Mannose & $3,9 \cdot 10^{-5}$ & $8,0 \cdot 10^{-5}$ \\
Fructose & $2,9 \cdot 10^{-3}$ & $6,7 \cdot 10^{-3}$ \\
\hline
\end{tabular}

Tab. 5. M i c h a e l is - Konstanten der Fraktionen I und II der Antennendrüse für drei Substrate.

27 Zit. nach H. NEtTER, Theoretische Biochemie, Springer, Berlin-Göttingen-Heidelberg 1959.

28 M. F. Krahl, A. K. Keltch, C. P. Walters and G. H. GLowes, J. gen. Physiol. 38, 31 [1954].

29 P. J. C. Sмгтн, Biochem. J. 76, 514 [1960]. und Fructose wurde die Reaktionsgeschwindigkeit im optischen Test bei 6 bis 8 verschiedenen Substratkonzentrationen bestimmt. Die gemessenen Werte wurden im graphischen Verfahren nach LineweAver und Burk ${ }^{27}$ ausgewertet. Die ermittelten $\mathrm{M}$ i c h a e $\mathrm{l}$ is - Konstanten sind in Tab. 5 zusammengefaßt.

\section{Diskussion und vergleichende Betrachtung}

Die drei untersuchten Organe des Flußkrebses enthalten übereinstimmend mindestens zwei Hexokinasen unterschiedlicher Spezifität. Die eine von ihnen, Fraktion II, kann man als unspezifische Hexokinase bezeichnen. Sie setzt die gleichen Zucker um, die auch von anderen unspezifischen Hexokinasen phosphoryliert werden, und die relativen Umsatzraten für die einzelnen Zucker stimmen weitgehend mit denen anderer Hexokinasen überein ${ }^{3,5,28-32}$. Die

\footnotetext{
30 A. Sols, Biochim. biophysica Acta [Amsterdam] 19, 144 [1956].

31 M. Ruiz-Amil, J. Insect. Physiol. 8, 259 [1962].

32 A. Hernández and A. Sols, Biochem. J. 86, 166 [1963].
} 
Übereinstimmung zeigt sich weiterhin auch in der Natur der Endprodukte der Phosphorylierung. Alle Zucker werden in C-6-Stellung phosphoryliert, wie dies auch für unspezifische tierische und pflanzliche Hexokinasen gilt. Es besteht natürlich noch die Mög. lichkeit, daß sich die Aktivität der hier als Fraktion II beschriebenen Hexokinase aus den Aktivitäten mehrerer Enzyme zusammensetzt, deren Trennung nicht gelungen ist. Nach dem chromatographischen Verhalten und der weitgehenden Übereinstimmung ihrer Eigenschaften mit bekannten Hexokinasen ist aber sehr wahrscheinlich, daß es sich um die weitverbreitete Form der unspezifischen Hexokinase handelt.

Das zweite isolierte Enzym, Fraktion I, das von den untersuchten Zuckern nur Mannose und Fructose umsetzt, darf man wohl zu den spezifischen Hexokinasen rechnen, da es bevorzugt Mannose phosphoryliert. $\mathrm{Da}$ außerdem noch eine geringere Aktivität mit Fructose besteht, widerspricht dem nicht, da fast alle als spezifische Hexokinasen beschriebenen Enzyme nicht nur einen Zucker umsetzen. Eine Ausnahme bilden hier anscheinend die vier streng spezifischen Enzyme des Hundebandwurmes ${ }^{10}$ und des Pärchenegels ${ }^{11}$, an denen allerdings nur fünf Zucker als Substrate getestet wurden. Tab. 6 gibt eine Über-

\begin{tabular}{|c|c|c|}
\hline Enzym & Zucker * & rel. Rate \\
\hline $\begin{array}{l}\text { Galaktokinase } \\
\text { aus Saccharo- } \\
\text { myces fragilis } \mathbf{3 6}\end{array}$ & $\begin{array}{l}\text { Galaktose } \\
\text { Galaktosamin } \\
\text { 2-Desoxygalaktose } \\
\text { Talose }\end{array}$ & $\begin{array}{l}1,0 \\
0,4 \\
0,4 \\
0,1\end{array}$ \\
\hline $\begin{array}{l}\text { Fructo-1-kinase } \\
\text { der Mucosa } \\
\text { (Ratte) }{ }^{37}\end{array}$ & $\begin{array}{l}\text { Fructose } \\
\text { Sorbose }\end{array}$ & $\begin{array}{l}1,0 \\
0,5\end{array}$ \\
\hline $\begin{array}{l}\text { Fructo-6-kinase } \\
\text { aus Erbsen }{ }^{38}\end{array}$ & $\begin{array}{l}\text { Fructose } \\
\text { Sedoheptulose } \\
\text { Glucose } \\
\text { Mannose }\end{array}$ & $\begin{array}{l}1,0 \\
0,2 \\
0,08 \\
0,06\end{array}$ \\
\hline $\begin{array}{l}\text { Fraktion I } \\
\text { aus Orconectes } \\
\text { limosus }\end{array}$ & $\begin{array}{l}\text { Mannose } \\
\text { Fructose }\end{array}$ & $\begin{array}{l}1,0 \\
0,22\end{array}$ \\
\hline
\end{tabular}

Tab. 6. Spezifitätsmuster einiger spezifischer Hexokinasen. * Es wurden hier nur die Zucker tabelliert, die als Substrate dienen. Die Umsatzrate des Hauptsubstrates wurde gleich 1 gesetzt.

sicht über einige als spezifische Hexokinasen beschriebene Enzyme. Man sieht, daß neben dem Hauptsubstrat auch geringere Aktivitäten mit ande-

33 D. S. Hoare and M. Kerly, Biochem. J. 58, 38 [1954]. ren Zuckern gefunden wurden. Die als Fraktion I bezeichnete Hexokinase aus Orconectes limosus paßt sich ohne Schwierigkeiten in diese Tabelle ein. Die Möglichkeit, daß auch bei Fraktion I ein Gemisch aus zwei spezifischen Enzymen vorliegt, ist natürlich nicht ganz von der Hand zu weisen. Auf Grund ihres chromatographischen Verhaltens ist die Fraktion I jedenfalls einheitlich. Eine Fructokinase, wie sie in der Leber der Säugetiere vorliegt, dürfte nicht vorhanden sein, da das Reaktionsprodukt Fructose-6Phosphat ist, während das Enzym der Leber in C-1Stellung phosphoryliert.

Vergleicht man die Mengen an Fraktion I und II in den einzelnen Organen, so ergibt sich das in Tab. 7 zusammengefaßte Bild. Als Maß für die

\begin{tabular}{lccc}
\hline \multicolumn{1}{c}{ Organ } & \multicolumn{2}{c}{ Gesamtaktivität $[\mathrm{nMol} / \mathrm{min}]$} & $\begin{array}{c}\text { Verhältnis } \\
\text { Fraktion I: }\end{array}$ \\
& Fraktion I & Fraktion II & \begin{tabular}{c} 
Fraktion II \\
\hline \multirow{2}{*}{ Antennendrüse }
\end{tabular} \\
& 4261 & 2975 & $1: 0,70$ \\
\multirow{2}{*}{ Enddarm } & 328 & 4420 & $1: 1,01$ \\
Herz & 3444 & 5040 & $1: 0,68$ \\
& 248 & 2115 & $1: 1,46$ \\
\hline
\end{tabular}

Tab. 7. Vergleich der Gesamtaktivitäten gegenüber Mannose in den Fraktionen I und II verschiedener Organe.

Menge an Enzym sind hier die Gesamtaktivitäten gegenüber Mannose in Fraktion I und II verglichen. Es zeigt sich, daß die Antennendrüse mehr spezifische Hexokinase besitzt als Enddarm und Herz. Das Mengenverhältnis für die Antennendrüse differiert in den vier Versuchen. Dies könnte seine Ursache in einer biologischen Variabilität, aber auch in der unterschiedlich raschen Inaktivierung der einzelnen Enzympräparationen haben. Letztere dürfte auch die Ursache dafür sein, daß sich bei Spezifitätsuntersuchungen an Rohextrakten kein eindeutiger Hinweis auf das Vorliegen der beiden Enzyme zeigte. Die hohe Instabilität mancher Hexokinasen ist auch aus anderen Untersuchungen bekannt ${ }^{6,29,33,34}$.

Welche Bedeutung das als Fraktion I bezeichnete spezifische Enzym im Stoffwechsel des Flußkrebses einnimmt, läßt sich nicht sagen. Spezifische Mannokinasen wurden bei Echinococcus granulosus ${ }^{10}$ und Schistosoma mansoni ${ }^{11}$ isoliert. Einen Hinweis für das Vorliegen eines besonderen Enzyms für die

34 E. WalaAs and O. W AlaAs, Acta chem. scand. 16, 1682 [1962]. 
Phosphorylierung der Mannose fanden Palleroni und Mitarbb. ${ }^{35}$ in Extrakten von Pseudomonas saccharophila. Ob der hohe Mannoseumsatz durch das Enzym des Flußkrebses in vivo überhaupt eine Rolle spielt, wird sich erst entscheiden lassen, wenn Näheres über den Kohlenhydratstoffwechsel des Versuchs-

35 N. J. Palleroni, R. Contopoulou, and M. Doudoroff, J. Bacteriol. 71, 202 [1956].

${ }^{36}$ F. Alvarado, Biochim. biophysica Acta [Amsterdam] 41, 233 [1960]. tieres bekannt ist. Möglicherweise ist überhaupt die Fructose das natürliche Substrat des spezifischen Enzyms.

Herrn Prof. Dr. K. URICH danke ich für Anregung und Förderung der Arbeit.

37 E. Cadenas and A. Sols, Biochim. biophysica Acta [Amsterdam] 42, 490 [1960].

38 A. Medina and A. Sols, Biochim. biophysica Acta [Amsterdam] 19, 378 [1956].

\title{
The epicuticle of millipedes belonging to the genera Cingalobolus and Aulacobolus with special reference to seasonal variations
}

\author{
G. Sundara Rajulu * and G. Krishnan
}

Department of Zoology, University of Madras, India

(Z. Naturforsch. 23 b, 845-851 [1968] ; eingegangen am 20. November 1967)

\begin{abstract}
The diplopods Cingalobolus bugnioni and Aulacobolus excellens possess an epicuticle formed of lipoproteins. But unlike in insect cuticles, the epicuticle lacks an outer lipid layer. The epicuticle is found only in the animals collected in summer months. At the commencement of the rainy season the new cuticle that is formed following moulting is without an epicuticle. The periodical presence of the epicuticle is of ecological significance. A study of the transpiration rates using animals with and without an epicuticle showed that in the former evaporation of water through the cuticle was much less than in the latter. It is suggested that the presence of an epicuticle during summer season is of adaptive value in that the restricted permeability properties of the epicuticle enable the animals to conserve their water resources. In the rainy season when there is plentiful supply of water in the environment the animals are without an epicuticle as there is no necessity to restrict water loss. The periodical absence of the epicuticle has been discussed in the light of previous reports on the cuticular organization of millipedes.
\end{abstract}

The cuticle of millipedes has not hitherto received much attention. What little we know about it is vitiated by the contradictory evidences brought forward by previous workers specially in regard to the existence of an epicuticle (Lagner ${ }^{1}$, CloudsleyThompson $^{2}$, Blower $^{3}$ ). Lagner ${ }^{1}$ noted in the cuticle of millipedes studied by her, a colourless layer external to the pigmented exocuticle which she suggested may be homologous to the epicuticle of insects. But Blower ${ }^{3}$ from studies on Tachipodoiulus niger and Schizophyllum sabulosum reported the absence of an epicuticle. He thought the colourless layer considered the epicuticle by earlier workers may be an optical effect and in support of this view pointed out that there was no outer nonchitinous cuticular layer corresponding to the insect epi-

* Present address: Lecturer in Zoology, Thiagarajar College, Madurai, India. - The paper includes results incorporated in a thesis submitted by G. Sundara Rajulu and approved for $\mathrm{Ph}$. D. degree of Madras University.

1 E. Langner, Zool. Jb. Abs. Anat. 63, 483 [1937]. cuticle. On the contrary, Cloudsley-Thompson ${ }^{2}$ distinguished in millipedes such as Paradesmus gracilis a colourless epicuticle, which may be separated by its differential solubility in chlorated nitric acid. But very little is known of the structure and composition of the epicuticle of millipedes.

Previous work indicates that in arthropods so far studied epicuticle may be constituted of two or more sub layers. Richards and Anderson ${ }^{4}$ from electron microscopic studies showed that the epicuticle of insects was formed of an outer lipid and inner lipoprotein layers. In insects like Rhodnius prolixus, Tenebrio molitor, Periplaneta americana, larva of Diataraxia oleracea and arachnids such as Tegenaria domestica. Ornithodorus moubata the epicuticle is three or four layered comprising an outermost

2 J. L. Cloudsley-Thumpson, Nature [London] 165, 692 [1950].

3 J. G. Blower, Quart. J. microscop. Sci. 92, 141 [1951].

4 A. G. Richards and T. F. Anderson, J. Morphology 71 , 135 [1952]. 